\title{
Predictors of Patient Satisfaction with the Feedback After a Neuropsychological Assessment
}

\author{
Ylva Holst ${ }^{*}, 1$, Håkan Nyman ${ }^{2}$ and Jan-Olov Larsson ${ }^{1}$ \\ ${ }^{I}$ Department of Woman and Child Health, Karolinska Institutet, Sweden \\ ${ }^{2}$ Håkan Nyman, Department of Clinical Neuroscience, Karolinska Institutet, Sweden
}

\begin{abstract}
An increasing number of adults in general psychiatry are referred for assessment regarding AttentionDeficit/Hyperactivity Disorders (ADHD) and Autism Spectrum Disorders (ASD). The aim of this study was to investigate patients' recollections of satisfaction with the feedback after a neuropsychological assessment, measured by the Assessment Questionnaire (AQ). The results showed that the patients generally had a low level of satisfaction with this feedback, and a low level of satisfaction was especially related to low self-esteem. The results indicate a need for more empirical studies of how assessments should be designed in order to increase satisfaction.
\end{abstract}

\section{INTRODUCTION}

The aim of this study was to investigate adult patients' recollections of satisfaction with the information/feedback given after a neuropsychological assessment, among patients with preliminary diagnoses of Attention-Deficit/Hyper-activity Disorder (ADHD; American Psychiatric Association [DSM-IVTR], 2000) and Autism Spectrum Disorders (ASD) [1]. These are important diagnoses to consider in general psychiatry [2,3]. $\mathrm{ADHD}$, for instance, is no longer regarded mainly as a childhood diagnosis in boys, but is now seen as a disorder affecting adults of both sexes [4, 5]. There is an increasing awareness of these disorders, and an increasing number of adults is referred for neuropsychological assessments.

In the Nordic countries, many outpatient clinics specialize in neuropsychological assessments. It seems that there are a substantial number of adults with these problems who were not diagnosed in childhood despite a high degree of contact with child psychiatric services [2]. It has been argued that these patients need a specialized neuropsychological assessment in order to evaluate their patterns of impairment [6]. After the assessment, most patients need continuing psychiatric help, proper medication, help with studies, and adaptations at work. However, support and treatment needs are not always met, especially for those patients with ADHD [6,7].

It has been proposed that the neuropsychological assessment itself is very important for the patients' selfesteem and well-being [8]. The idea is that patients who have struggled throughout their lives with neuropsychological and social problems would benefit from going through a neuropsychological assessment and receiving an explanation for their psychiatric and cognitive impairments. "Even among clients with significant neurological impairment,

*Address correspondence to this author at the Department of Woman and Child Health, Karolinska Institutet, Sweden; Tel: +46(8)6866608;

E-mail: ylva.holst@bredband.net feedback might be an important step in the assessment process in which the psychologist is able to provide information that is vital to clients' self-understanding and ability to plan for their futures" [9] (p. 311).

Providing feedback that preserves patients' self-esteem is crucial in a psychiatric setting [10]. Children with ADHD have been found to have low self-esteem [11]. However, less is known about adult patients and how self-esteem is influenced by receiving an ADHD diagnosis in adulthood [12]. A study of ADHD from childhood to adulthood found that adults also suffer from poor self-esteem [13]. The relation between ASD and self-esteem has not been studied to the same extent as that between ADHD and self-esteem. It seems that people with autism often have negative life experiences due to other "non-autistic" people who do not understand how to talk to and listen to autistic people [14].

Historically, clinicians began sharing assessment results with patients more regularly due to recognition of patients' legal rights to access professional records $[15$, \{Gass, 1992 \#200 \} 16]. The focus was also broadened from test measures to the patient-examiner relationship, the patients' difficulties, and the examiners' counter transference. Additionally, clinicians discovered that patients benefited from feedback in many ways; their self-esteem and feelings of hope increased, while feelings of isolation and symptomatology decreased [17]. Some clinicians began to write about positive patient changes during assessment. These changes seemed to occur when patients were included in the assessment process as active participants [18-20].

McGrath (2001) emphasizes that assessment is not useful if individuals receiving the feedback do not find the results interesting or useful, no matter how valid the results are. The results of an informal methodological review of 108 articles published during one year in three major assessment journals (Assessment, Journal of Personality Assessment, \& Psychological Assessment) revealed a complete lack of any studies evaluating how test takers felt about the assessment [21]. 
The aim of the present study is therefore to describe how patients with neuropsychiatric problems experience the assessment process and the feedback from the examiner, and to investigate the relationship between this and a number of patient characteristics including self-esteem, physical and mental health, sex, and personality disorders. The regional ethical committee in Stockholm, Karolinska Institutet, reviewed and approved the study on 27 September 2005, (2005/1057-32).

\section{MATERIAL AND METHODOLOGY}

\section{Participants}

The patients were recruited from two psychiatric outpatient clinics in the Stockholm area with teams specialized in the assessment of ADHD and AS. The time they had spent on the waiting list ranged from 3 months to 26 months, with a mean of 13 months.

Demographic data about the 32 participants were collected from their medical records: 17 men and 15 women, average age 36 years (range 20-56). The neuropsychological assessment resulted in the following diagnoses: ADHD $(\mathrm{n}=$ $11)$, ASD $(n=15)$, Tourette's syndrome $(n=1)$, and mental retardation $(n=3)$. The remaining two patients did not fulfill the criteria for a diagnosis. A majority of the patients were living with a partner and had children. The mean level of education was 11 years. Sixteen patients were receiving social assistance, were sick-listed, or were on social welfare. Twenty-eight had a previous psychiatric contact. Ten had at least one relative with diagnosed neurodevelopmental disorder or related symptoms. Twenty-two patients had comorbid disorders with psychiatric symptoms. Twelve had an identified somatic disorder such as epilepsy or migraine. Nineteen were taking psychotropic drugs. Complete demographic data are provided.

\section{Procedures}

The assessments were performed by ten psychologists, in cooperation with physicians. Patients assessed during 2004 were consecutively invited to participate (clinic $1: \mathrm{n}=30$, clinic 2: $n=29)$. Thirty-two patients $(55.9 \%)$ gave written consent to participate in the study, 17 men and 15 women. Twenty-four patients had been referred, and the remaining eight patients had contacted the clinics on their own initiative. All patients had participated in a neuropsychological assessment.

A letter with information about the study was sent to the patients together with the questionnaires and a pre-stamped envelope for the return of the questionnaires. The patients were instructed to return the questionnaires within 1 month. The patients who did not return the questionnaires were sent a reminder including a new questionnaire and a pre-stamped envelope.

Patients who found it difficult to answer the questionnaires had the option of receiving personal help from the first author. Three of the participating patients found it difficult to answer the questionnaires; two of them chose to visit the clinic for assistance, while the other preferred to be helped via telephone. The data collection and analysis of the questionnaires and the medical records took place between May 2005 and January 2006.

\section{The Neuropsychological Assessment}

Neuropsychological assessments are designed to collect information about patients whose mental symptoms are thought to be related to brain dysfunction [22]. The assessment is performed in multiple steps. Usually, it is designed as an information-gathering process which begins with an anamnestic interview with the patient and a close relative, generally the mother, in order to obtain a complete developmental history. Previous assessments, medical records, and other documents of interest are collected and reviewed. A comprehensive battery of neuropsychological tests is administered to the patient. The assessment is performed by a psychologist trained in neuropsychology in cooperation with a physician. Sometimes additional neuroimaging techniques are needed, and other professionals such as neurologists are consulted if necessary. During and after the assessment, the patient receives various types of feedback from the examiner according to the routines at the outpatient clinic and the individual needs of the patient. The psychologist writes a report describing the outcome of the assessment concerning memory, concentration, attention, language, verbal fluency, and problem solving. Feedback is usually delivered information given by the psychologist reading the report together with the patient in order to emphasize strengths and problem areas, and to explain difficult words and concepts. The patient always receives a copy of the report.

\section{Measures}

The patients' experiences, and their satisfaction with the feedback after the assessment, were measured with the Assessment Questionnaire (AQ) [23]. Self-administered questionnaires were used to measure self-perceived health (SF-36, GAF), personality disorders (DIP-Q), and basic and earning self-esteem. Medical records were scrutinized for socio-demographic data, with the exception of one patient who did not give permission for this. One patient did not complete the AQ.

\section{The Assessment Questionnaire (AQ)}

The AQ consists of 48 Likert scale items generating four first-order factors: 1) New Self-awareness/Understanding, 2) Positive Accurate Mirroring, 3) Positive Relationship with the Examiner, and 4) Negative Feelings about the Assessment. The subscales are summed up in an overall measure of Total Satisfaction [24]. In the present study, internal reliability for the four subscales, measured with Cronbach's Alpha, varied between 0.80 and 0.94 .

\section{SF-36}

Self-perceived health was measured by the SF-36 [25], which includes eight subscales: 1) Physical Functioning, 2) Role-Physical, 3) Bodily Pain, 4) General Health, 5) Vitality, 6) Social Functioning, 7) Role-Emotional, and 8) Mental Health. 
The SF-36 health survey was developed as a general measure with a broad usage [25]. Apart from the eight subscales, two additional dimensions (sum indices) are calculated, Physical Component Score (PCS) and Mental Component Score (MCS). PCS consists of the subscales: 1) Physical Functioning, 2) Role-Physical, 3) Bodily Pain, and 4) General Health. MCS consists of the subscales: 5) Vitality, 6) Social Functioning, 7) Role-Emotional and 8) Mental Health.

\section{Global Assessment of Functioning (GAF)}

The Global Assessment of Functioning (GAF) rates psychological functioning on a scale of 1-100, where 51-60 is considered to reflect moderate symptoms [26]. The scale is aimed at measuring mental, social, and vocational functioning in global terms. It was developed from the Health-Sickness Rating Scale [27] and is now included in DSM-IV.

\section{DIP-Q}

Personality disorders were screened for with the DSM-IV and ICD-10 Personality Questionnaire (DIP-Q) [28, 29]. This instrument is a self-assessment scale. The construction of the statements was derived from the diagnostic criteria of DSM-IV and ICD-10. A self-assessed GAF over 70 makes a personality disorder diagnosis less probable. DIP-Q has been shown to have a good test-retest-reliability for all DSM-IV personality disorders, if patients with depression or anxiety symptoms are excluded [30].

A study compared three psychiatric samples in order to validate the self-report questionnaire DIP-Q. Results showed that the odds ratio for an Axis II disorder was nearly five times higher among psychiatric patients, independent of concomitant Axis I disorders, gender or age [31].

\section{Basic and Earning Self-Esteem Scales}

Self-esteem was measured with a questionnaire measuring two aspects of self-esteem, basic and earning selfesteem [32, 33]. The Basic Self-Esteem Scale was developed to capture self-acceptance independent of external attributes and competence. The questions reflect the subjects' inner attitudes towards themselves and others, and their attitudes towards life in general. The Earning Self-Esteem Scale is aimed at capturing the conditions attached to self-esteem; the striving for success, competence, and perfection by making hard demands on oneself, and being in control of oneself and others in order to attain these goals.

In order to construct- and cross-validate the scales, they were related to other well-known personality scales as well as to a projective technique (TAT) in two independent samples of first semester psychology students $(n=153, n=$ 82). Basic SE showed concurrent validity being highly correlated $\quad(p<0.001)$ with both Rosenberg's and Coopersmith's self-esteem scales and highly or moderately with Neuroticism, Extraversion (EPQ) and Test-Anxiety (TAS) in both samples. Earning SE gained some construct validation by its relation to Type-A scores (JAS) as well as to verbal fantasy stories on TAT-pictures measuring Need of Achievement [32].

\section{Statistics}

Pearson correlation was used to identify relations between AQ scores and age, gender, diagnosis, GAF, SF-36, Dip-Q, and Basic and Earning Self-Esteem.

The statistically significant (defined as $\mathrm{p}<0.1$ ) variables General Health, Mental Health (SF-36), and Basic SelfEsteem were then included in a multivariate regression analysis with degree of satisfaction as the dependent variable. In all other analyses, $\mathrm{p} \leq 0.05$ was registered as statistically significant. A t-test was used to see if there were any significant differences regarding the four subscales in the AQ.

In order to further clarify the difference between the more satisfied and the less satisfied patients, we operationalized satisfaction/dissatisfaction by dividing the subjects into two groups according to the AQ median (> or < $3.4)$, yielding one group $(n=16)$ regarded as being "more satisfied" with the assessment and another group $(\mathrm{n}=16)$ regarded as being "less satisfied" with the assessment. Cronbach's alpha was computed for each of the four subscales to measure the reliability of the AQ. The data were analyzed with the SPSS software package (Statistical Package for the Social Sciences, version 13.0).

\section{RESULTS}

\section{Satisfaction with Feedback (AQ)}

A t-test showed that the total study group scored low on the subscales of New Self-Awareness $(p=0.000)$ and Positive/Accurate Mirroring $(\mathrm{p}=0.013)$.

The two groups differed significantly on Total Satisfaction $(\mathrm{p}=0.013)$. The less-satisfied group reported low satisfaction with New Self-Awareness $(p=0.045)$ and scored high on Negative Feelings $(p=0.003)$.

\section{Self-Perceived Health (SF-36)}

Overall mental health according to the Mental Component Score (MCS) was worse in the study group ( $\mathrm{p}=$ $<0.001$ ) than in a norm group drawn from the general Swedish population [25]. Overall physical health, measured by the Physical Component Score, did not differ $(\mathrm{p}=0.560)$.

The more satisfied patients reported better physical health $(\mathrm{p}=0.008)$ and also better mental health $(\mathrm{p}=0.028)$ than the less satisfied patients (Table 1).

\section{GAF}

There were no significant differences between the less satisfied and more satisfied groups regarding functioning during the previous year or the previous weeks (Table 1).

\section{Personality Disorders (DIP-Q)}

Thirty patients had at least one personality disorder according to DIP-Q (range 1-9). The most frequently reported indications were from Avoidant, Obsessive Compulsive, and Borderline disorders. There was no significant difference between the more satisfied and the less satisfied patients (Table $\mathbf{1}$ ). 


\section{Self-Esteem}

The more satisfied patients reported higher basic selfesteem $(p=0.001)$ than the less satisfied patients, but the groups did not differ in terms of earning self-esteem (Table 1).

\section{AQ Related to Patient Characteristics}

The Pearson correlation analysis revealed statistically significant relationships between low AQ Total Satisfaction and poor general health $(\mathrm{SF}-36)(\mathrm{p}=0.008)$, poor mental health (SF-36) $(\mathrm{p}=0.028)$, and a low score on the Basic Self-Esteem scale $(\mathrm{p}=0.001)$. There were no statistically significant relationships between AQ Total Satisfaction and age, sex, or diagnosis (e.g. ADHD or ASD) (Table 1).

In the regression analysis using $\mathrm{AQ}$ as the dependent variable and General Health, Mental Health, and Basic SelfEsteem scale as independent variables, there was a significant relationship between low satisfaction and low self-esteem $(p=0.028)$ (Table 2$)$.

\section{DISCUSSION}

The aim of this study was to investigate patients' recollections of satisfaction with the feedback after a comprehensive neuropsychological assessment, and to relate the characteristics of the patients to this experience. When examining the patients' medical records, it became obvious that a considerable amount of effort is put into neuropsychological assessments by committed professionals at the psychiatric clinics. The main findings were that on average the patients expressed rather low satisfaction with the feedback in relation to the assessment, and that a low level of satisfaction was related to low self-esteem.

We did not find any significant relationships between level of satisfaction and diagnosis, age, sex, or number of personality disorders. The patients who reported less satisfaction and low self-esteem also reported higher scores on the AQ subscale Negative Feelings, and lower scores on the New Self-Awareness/Understanding subscales in AQ. The more satisfied patients reported higher self-esteem; they also reported having felt confirmed by the examiner and

Table 1. Characteristics of Patients who were Satisfied/Dissatisfied with the Feedback After the Neuropsychological Assessment

\begin{tabular}{|c|c|c|c|}
\hline \multirow[t]{2}{*}{ Item } & \multicolumn{2}{|c|}{ Patients' Report About Feedback } & \multirow[t]{2}{*}{ p-Value } \\
\hline & Satisfied & Dissatisfied & \\
\hline Age m (sd) & $37(11.4)$ & $35(8.2)$ & 0.550 \\
\hline Sex & & & \\
\hline Male $(\mathrm{n}=17)$ & 7 & 10 & 0.303 \\
\hline Female $(\mathrm{n}=15)$ & 9 & 6 & \\
\hline \multirow{2}{*}{\multicolumn{4}{|c|}{$\begin{array}{l}\text { Diagnosis after } \\
\text { Assessment }(n=32)\end{array}$}} \\
\hline & & & \\
\hline ADHD & 7 & 4 & 0.309 \\
\hline Autism & 7 & 8 & \\
\hline Other diagnosis/no diagnosis & 2 & 4 & \\
\hline Physical Functioning m (sd) & $86.9(18.2)$ & $86.9(13.1)$ & 1.00 \\
\hline Role-Physical & $59.4(43.7)$ & $60.0(8.7)$ & 0.967 \\
\hline Bodily Pain & $63.9(27.19)$ & $66.3(31.2)$ & 0.817 \\
\hline General Health & $61.3(23.3)$ & $38.2(23.1)$ & 0.008 \\
\hline Vitality & $46.2(20.1)$ & $36.0(15.5)$ & 0.105 \\
\hline Social Functioning & $59.4(34.0)$ & $46.9(32.7)$ & 0.298 \\
\hline Role-Emotional & $50.0(43.9)$ & $37.8(45.2)$ & 0.451 \\
\hline Mental Health & $60.2(22.6)$ & $39.8(27.2)$ & 0.028 \\
\hline Physical Component Score (PCS) & $48.8(9.8)$ & $49.5(6.6)$ & 0806 \\
\hline Mental Component Score (MCS) & $34.8(14.4)$ & $24.2(14.4)$ & 0.051 \\
\hline $\begin{array}{l}\text { Personality } \\
\text { disorders (DIP-Q) }\end{array}$ & $3.3(1.8)$ & $4.6(2.5)$ & 0.111 \\
\hline Minimum & 1 & 0 & \\
\hline Maximum & 6 & 9 & \\
\hline Basic Self-Esteem m (sd) & $3.5(0.69)$ & $2.7(0.6)$ & 0.001 \\
\hline Earning Self-Esteem m (sd) & $3.8(0.4)$ & $3.7(0.6)$ & 0.776 \\
\hline
\end{tabular}


having been able to establish a positive relationship with the examiner according to the AQ subscale Positive Relationship. Our results may be interpreted as support for the suggestion that patients may become more satisfied and experience an increase in their self-esteem when they experience a positive and empathic relationship with the examiner [17, 19, 34].

Table 2. Relationships Between Satisfaction with Feedback (AQ) and Patient Characteristics. Multivariate Analysis

\begin{tabular}{|c|c|c|c|}
\hline & Beta & t & sign \\
\hline \hline Basic Self-Esteem & 0.453 & 2.319 & $\mathbf{0 . 0 2 8}$ \\
\hline General Health & 0.110 & 0.506 & 0.617 \\
\hline Mental Health & 0.106 & 0.535 & 0.598 \\
\hline
\end{tabular}

The patients' levels of satisfaction must be interpreted in the perspective of their psychiatric history. A majority of the patients in our study had had a previous psychiatric contact concerning other psychiatric diagnoses prior to being referred for an assessment.

The patients' had an average of 4 self-reported personality disorders, which is comparable to the results in a study of women with chronic eating disorders, where the median was 3 personality disorders [29], and to the results in a study of personality disorders in former child psychiatric patients, where the average was 1.7 personality disorders [28].

Taking part in a neuropsychological assessment may be very demanding for the patient. The results of the assessment may be extremely important and often have far-reaching consequences for the patient's whole life-situation and future. The assessment results are often the basis for important decisions about work, economic support, or entitlement to specific help interventions from the community. Low satisfaction may stem from disappointment with the results and the entire assessment procedure and/or an unsatisfying contact with the examiner.

The assessment process is perhaps not always optimal for patients with ADHD or ASD. It is important that the examiner conducting neuropsychological assessments has suitable training for this assignment, and also that he or she can give feedback that is specially designed for patients with neurodevelopmental disorders.

Earlier studies have shown that patients usually benefit in many ways from being more involved in their own examinations and assessments $[10,34,35-37]$. This particular group of patients might benefit even more by being involved in the assessment process.

In order to provide psychological assessment and feedback in a more systematic way, different methods have been used in treatment planning, as well as before or during mental health treatments [38, 39]. For example, in Therapeutic Assessment (TA) [36] it is important to work collaboratively with the patients in a very organized way before, during, and after the assessment [34]. TA was used in a study examining the interaction between therapeutic alliance and in-session process during the assessment phase of treatment. The study compared the utility of the TA model versus a traditional information gathering model of assessment. The results indicated that the use of a TA model may decrease the number of patients who terminate treatment against medical advice, and that the psychological assessment process may impact the patient's experience of assessment feedback and aid in the development of a therapeutic alliance [40].

Self-esteem can be low in patients with ADHD or ASD [11-13]. Patients with lower self-esteem may be more anxious and vulnerable in an assessment process, and need to be treated with more consideration. The results from one study measuring the consumer's satisfaction with neuropsychological assessments indicated that neuropsychologists should pay particular concern to the feedback and support they give to highly anxious clients [35].

The less satisfied patients reported a low basic selfesteem, which could indicate that these patients have had difficulty developing stable and satisfactory self-esteem during childhood, depending on different cognitive and mental impairments which in many ways might complicate a satisfactory personality development. They may have difficulties in making contact with others, and also difficulties in developing a trusting relationship with an examiner during an investigation. We believe that patients' satisfaction and self-esteem can be improved considerably by altering the way in which neuropsychological assessments are performed so as to show more consideration for the individual patient's perspective than is currently the case.

In summary, patients who had participated in a neuropsychological assessment expressed, on average, a rather low satisfaction with both the assessment and the subsequent feedback. The patients in the study group were less satisfied with positive and accurate mirroring, and did not think they had gained new knowledge about themselves. A low degree of satisfaction was strongly related to low selfesteem. The results of this study indicate a need for more empirical studies of how assessments should be designed to make patients feel more involved, and more satisfied with the assessment process.

\section{ACKNOWLEDGEMENTS}

We thank the staff and patients at The Neuropsychiatric Diagnostic Team in Liljeholmen and Psykiatrin Södra for participating in this study.

\section{REFERENCES}

[1] Frith U, Happe F. Autism spectrum disorder. Curr Biol 2005; 15(19): R786-90.

[2] Torgersen T, Gjervan B, Rasmussen K. ADHD in adults: A study of clinical characteristics, impairment and comorbidity. Nord J Psychiatry 2006; 60: 38-43.

[3] Nylander L, Gillberg C. Screening for autism spectrum disorders in adult psychiatric out-patients: a preliminary report. Acta Psychiatr Scand 2001; 103(6): 428-34. 
[4] Wilens TE, Kwon A, Tanguay S, et al. Characteristics of adults with attention deficit hyperactivity disorder plus substance use disorder: the role of psychiatric comorbidity. Am J Addict 2005; 14(4): 319-27.

[5] Spencer TJ, Biederman J, Mick E. Attention-deficit/hyperactivity disorder: diagnosis, lifespan, comorbidities, and neurobiology. Ambul Pediatr 2007; 7(Suppl 1): 73-81.

[6] Brar A, Flyckt L. Vuxna med neuropsykiatrisk diagnos får inte tillräcklig hjälp. Lakartidningen 2006; 103(19): 1516-22.

[7] Bejerot S. Excluded patient group. A new approach is necessary for adults with ADHD/Tourette syndrome/autism spectrum disorders. Lakartidningen 2006; 103(19): 1508.

[8] Bejerot S. Rehabilitation! Diagnosis can be a turning point for adults with ADHD/autism spectrum disorders. Lakartidningen 2004; 101(42): 3222-3.

[9] Smith SR, Wiggins CM, Gorske TT. A survey of psychological assessment feedback practices. Assessment 2007; 14(3): 310-9.

[10] Allen JG, Lewis L, Blum S, Voorhees S, Jernigan S, Peebles MJ. Informing psychiatric patients and their families about neuropsychological assessment findings. Bull Menninger Clin 1986; 50(1): 64-74.

[11] Edbom T, Lichtenstein P, Granlund M, Larsson JO. Long-term relationships between symptoms of attention deficit hyperactivity disorder and self-esteem in a prospective longitudinal study of twins. Acta Paediatr 2006; 95(6): 650-7.

[12] Slomkowski C, Klein RG, Mannuzza S. Is self-esteem an important outcome in hyperactive children? J Abnorm Child Psychol 1995; 23(3): 303-15

[13] Mannuzza S, Klein RG. Long-term prognosis in attention-deficit/ hyperactivity disorder. Child Adolesc Psychiatr Clin N Am 2000; 9(3): 711-26.

[14] Aylott J. Understanding and listening to people with autism. Br J Nurs 2001; 10(3): 166-72.

[15] Brodsky SL. Shared results and open files with the client. Prof Psychol 1972; 3: 362-4.

[16] Gass CS, Brown MC. Neuropsychological test feedback to patients with brain dysfunction. Psychol Assess 1992; 4(3): 272-7.

[17] Finn SE, Butcher JN, Eds. Clinical objective personality assessment. $2^{\text {nd }}$ ed. New York: Pergamon Press 1991.

[18] Berg M. The feedback process in diagnostic psychological testing. Bull Menninger Clin 1985; 49(1): 52-69.

[19] Fischer CT. Individualizing psychological assessment. New Jersey, Hillsdale: Lawrence Erlbaum 1994.

[20] Harrower M. Projective counseling - A psychotherapeutic technique. Am J Psychother 1956; 10: 74-86.

[21] McGrath RE. Toward more clinically relevant assessment research. J Pers Assess 2001; 77(2): 307-32.

[22] Berrios SE, Markova IS. Assessment and measurement in neuropsychiatry: a conceptual history. Semin Clin Neuropsychiatry 2002; 7(1): 3-10.

[23] Finn S, Schroeder DG, Tonsager M. The assessment questionnaire2 (AQ-2): a measure of clients' experiences with psychological assessment. Austin, Texas: Center for Therapeutic Assessment 1993.

[24] Finn SE, Bunner MR. Impact of test feedback on psychiatric inpatients' satisfaction with assessment. In the 28th Annual Symposium on Recent Developments in the use of the MMPI. St. Petersburg Beach FL 1993.

[25] Sullivan M, Karlsson J, Taft C. SF-36 Health Survey: Swedish Manual and Interpretation Guide. $2^{\text {nd }}$ ed. Gothenburg 2002.

[26] American Psychiatric Association. Diagnostic and statistical manual of mental disorders. $4^{\text {th }}$ ed. Washington DC 1994.

[27] Luborsky L. Clinician's judgments of mental health. Arch Gen Psychiatry 1962; 7: 407-17.

[28] Ramklint M, von Knorring AL, Ekselius I. Personality disorders in former child psychiatric patients. Eur Child Adolesc Psychiatry 2002; 11(6): 289-95.

[29] Larsson JO, Hellzen M. Patterns of personality disorders in women with chronic eating disorders. Eat Weight Disord 2004; 9(3): 2005 .

[30] Ottosson H, Bodlund O, Ekselius I, et al. The DSM-IV and ICD-10 personality questionnaire (DIP-Q) construction and preliminary validation. Nord J Psychiatry 1995; 49: 285-91.

[31] Bodlund O, Grann M, Ottosson H, Svanborg C. Validation of the self-report questionnaire DIP-Q in diagnosing DSM-IV personality disorders: a comparison of three psychiatric samples. Acta Psychiatr Scand 1998; 97(6): 433-9.

[32] Forsman L, Johnson M. Dimensionality and validity of two scales measuring different aspects of self-esteem. Scand J Psychol 1996; 37: $1-15$.

[33] Johnson M. Self-esteem stability: the importance of basic selfesteem and competence for the stability of global self-esteem. Eur J Pers 1998; 12: 103-16.

[34] Finn SE, Tonsager ME. Therapeutic effects of providing MMPI-2 test feedback to college students awaiting therapy. Psychol Assess 1992(4): 278-87.

[35] Bennett-Levy J, Klein-Boonschate MA, Batchelor J, McCarter R, Walton N. Encounters with Anna Thompson: the consumer's experience of neuropsychological assessment. Clin Neuropsychol 1994; 8(2): 219-38.

[36] Finn SE. Inormation-gathering and therapeutic models of assessment: complementary paradigms. Psychol Assess 1997; 9(4): 374-85.

[37] Hanson WE, Claiborn CD, Kerr B. Differential effects of two testinterpretation styles in counseling: a field study. J Couns Psychol 1997; 44(4): 400-5.

[38] Haynes SN, Leisen MB, Blaine DD. The design of individualized behavioral treatment programs using functional analytic case models. Psychol Assess 1997; 9: 334-48.

[39] Harkness AR, Lilienfeld SO. Individual differences science for treatment planning: personality traits. Psychol Assess 1997; 9: 34960 .

[40] Ackerman S, Hilsenroth MJ, Baity MR, Blagys MD. Interaction of therapeutic process and alliance during psychological assessment. J Pers Assess 2000; 75(1): 82-109. 